\title{
Boundary element method for micropolar fluid flow in a channel
}

\author{
M. Zadravec, M. Hriberšek \& L. Škerget \\ University of Maribor, Faculty of Mechanical Engineering, Slovenia
}

\begin{abstract}
The contribution deals with numerical simulation of micropolar fluid flow in channel, describing flow of suspensions with rigid and underformable particles with own rotation. The micropolar fluid flow theory is incorporated into the framework of a velocity-vorticity formulation of Navier-Stokes equations. The governing equations are derived in differential and integral form, resulting from the application of a Boundary Element Method (BEM). In integral transformations, the diffusion-convection fundamental solution for flow kinetics, including vorticity transport, heat transport and microrotation transport, is implemented. The Poiseuille flow test case is the benchmark case of channel flow. The results show, that the ratio between vortex viscosity coefficient and spin gradient viscosity coefficient controls the microrotation in the micro channel.
\end{abstract}

Keywords: boundary element method, micropolar fluid, poiseuille flow.

\section{Introduction}

In the recent years there was a progress in a micromachining technology. Opinion of a few scientists is that flows on the microscale differ from that on a macroscale, described by the Navier-Stokes equations. To clarify such assumption micropolar fluid theory is gaining interest of a lot of researchers. Micropolar fluids are a subclass of microfluids, introduced by Eringen [1]. A simple microfluid is by Eringen's definition a fluid medium whose properties and behaviour are influenced by the local motions of the material particles contained in each of its volume elements. A microfluid is isotropic viscous fluid and possesses local inertia. Because of a complex formulation for a general microfluid this class of fluids is divided into subclasses, which allow a simplified 
description of the effects arising from particle micromotions. As mentioned, in micropolar fluids, a subclass of microfluids, rigid particles contained in a small volume element can rotate about the center of the volume element, which is described by the micro-rotation vector (Eringen [1,2]). This local rotation of the particles is independent of the mean fluid flow and its local vorticity field. Lukaszewicz [3] presented in his book mathematical aspects of the micropolar fluid flow theory. From this theory it is also expected to successfully describe non-Newtonian behaviour of certain fluids, such as liquid crystals, ferro liquids, colloidal fluids, liquids with polymer additives, animal blood carrying deformable particles (platelets), clouds with smoke, suspensions, slurries and liquid crystals. This theory gain attention of researchers in recent years also in other fields of fluid flows.

Papautsky et al [4] described microchannel fluid flow behaviour with numerical model based on micropolar fluid theory and experimentally verified the model. Results showed that micropolar fluid theory present better agreement with experiment than the use of classical Navier-Stokes theory. In the paper of Pietal [5] it is evident that applicability of the micropolar fluid theory in microchannels depends on the geometrical dimension of the flow field.

Among different approximation methods for solving problems of fluid flow the Boundary Element Method (BEM) is a relatively new method with some interesting features, described in Škerget et al [6,7], Hriberšek and Škerget [8]. Here, we will focus on the development of BEM for velocity-vorticity formulation of Navier-Stokes equations presented by Škerget et al [6] and show how to incorporate the micropolar fluid theory into the BEM framework.

\section{Mathematical formulation}

For description of compressible viscous fluid flow we use conservation laws for mass, momentum and energy with appropriate rheological models and equations of state. In the case of isothermal fluids which behaviour can be described by micropolar fluid flow theory Eringen [1] presented modified equation of conservation laws for mass (1), momentum (2) and microrotation (3):

$$
\begin{gathered}
\frac{\partial \rho}{\partial t}+\vec{\nabla}(\rho \vec{v})=0 \\
\rho \frac{D \vec{v}}{D t}=-\vec{\nabla} p+\left(\lambda_{v}+2 \mu_{v}+k_{v}\right) \vec{\nabla} \vec{\nabla} \cdot \vec{v}- \\
-\left(\mu_{v}+k_{v}\right) \vec{\nabla} \times \vec{\nabla} \times \vec{v}+k_{v} \vec{\nabla} \times \vec{N}+\rho \vec{f} \\
\rho j \frac{D \vec{N}}{D t}=\left(\alpha_{v}+\beta_{v}+\gamma_{v}\right) \vec{\nabla} \vec{\nabla} \cdot \vec{N}-\gamma_{v} \vec{\nabla} \times \vec{\nabla} \times \vec{N}+k_{v} \vec{\nabla} \times \vec{v}-2 k_{v} \vec{N}+\rho \vec{l}
\end{gathered}
$$

Differential operator $D(\cdot) / D t=\partial(\cdot) / \partial t+v_{k} \partial(\cdot) / \partial x_{k}$ represents the Stokes material derivative. In the next step we assume that fluid mass density $\rho$ and all 
micropolar fluid properties as second order viscosity coefficient $\lambda_{v}$, dynamic viscosity $\mu_{v}$, vortex viscosity coefficient $k_{v}$, viscosity gradient coefficients $\alpha_{v}, \beta_{v}, \gamma_{v}$ and microinertia $j$, are constant parameters. We also consider zero couples $\vec{f}$ and $\vec{l}$. Therefore we can rewrite equations (1) - (3) for the assumption that micropolar fluid flow will be viscous, incompressible, steady state and laminar also using vector algebra $(\vec{\nabla} \times \vec{\nabla} \times \vec{F}=\vec{\nabla}(\vec{\nabla} \cdot \vec{F})-\Delta \vec{F})$. Considering the mass conservation and accounting for the solenoidality of microrotation field the mass, momentum and microrotation conservation equations are further simplified to:

$$
\begin{gathered}
\vec{\nabla} \cdot \vec{v}=0 \\
\rho \frac{D \vec{v}}{D t}=-\vec{\nabla} p+\left(\mu_{v}+k_{v}\right) \Delta \vec{v}+k_{v} \vec{\nabla} \times \vec{N} \\
\rho j \frac{D \vec{N}}{D t}=\gamma_{v} \Delta \vec{N}+k_{v} \vec{\nabla} \times \vec{v}-2 k_{v} \vec{N}
\end{gathered}
$$

To incorporate micropolar fluid flow theory into the framework of velocityvorticity formulation of Navier-Stokes equations and to apply the BEM approximation method, we must first split the dynamics of the flow into its kinematic and kinetic part. This is done by the use of derived vector vorticity field function $\vec{\omega}$, obtained as a curl of the compatibility velocity field $\vec{\omega}=\vec{\nabla} \times$ $\vec{v}$, which is solenoidal vector function by the definition $\vec{\nabla} \cdot(\vec{\nabla} \times \vec{v})=0$. By applying the curl operator to vorticity and using the mass conservation equation (5) for the incompressible fluid flow we get elliptic Poisson equation for the velocity vector, Skerget et al [6]:

$$
\Delta \vec{v}+\vec{\nabla} \times \vec{\omega}=0
$$

or in tensor notation form:

$$
\frac{\partial^{2} v_{i}}{\partial x_{j} \partial x_{j}}+e_{i j k} \frac{\partial \omega_{k}}{\partial x_{j}}=0
$$

Equation (8) represents kinematic part of the fluid flow where for known vorticity field, the corresponding velocity field can be determined.

To compute the kinetic part of the flow we apply the curl operator to the momentum conservation equation (5) and considering that $\vec{\nabla} \cdot \vec{\omega}=0, \vec{\nabla} \cdot \vec{v}=0$ and $\vec{\nabla} \cdot \vec{N}=0$ due to the vorticity and microrotation definition and mass conservation equation, it follows:

$$
\rho \frac{D \vec{\omega}}{D t}=\left(\mu_{v}+k_{v}\right) \Delta \vec{\omega}+(\vec{\omega} \cdot \vec{\nabla}) \vec{v}-k_{v} \Delta \vec{N}
$$


This equation shows that the rate of change of the vorticity field is due to viscous diffusion, vortex stretching and twisting, and microrotation.

For the case of two-dimensional plane flow and accounting for all previous assumption the final form of equations for kinematic and kinetic part is expressed in Cartesian tensor notation form as:

$$
\begin{gathered}
\frac{\partial^{2} v_{i}}{\partial x_{j} \partial x_{j}}+e_{i j} \frac{\partial \omega}{\partial x_{j}}=0 \\
\rho \frac{D \omega}{D t}=\left(\mu_{v}+k_{v}\right) \frac{\partial^{2} \omega}{\partial x_{j} \partial x_{j}}-k_{v} \frac{\partial^{2} N}{\partial x_{j} \partial x_{j}} \\
\rho j \frac{D N}{D t}=\gamma_{v} \frac{\partial^{2} N}{\partial x_{j} \partial x_{j}}+k_{v} e_{i j} \frac{\partial v_{i}}{\partial x_{j}}-2 k_{v} N
\end{gathered}
$$

If we assume that $k_{v}=0$ the equation for vorticity (11) and microrotation (12) are uncoupled, therefore the flow is independent of the microrotation, and the governing equations now resume the form of the classical Navier-Stokes equations.

When we use the velocity-vorticity formulation the pressure term is eliminated from momentum conservation equation (5). In order to compute pressure distribution we derive the equation for pressure, which is obtained from equation (5) with considering mass conservation equation (7):

$$
\vec{\nabla} p=-\rho \frac{D \vec{v}}{D t}-\left(\mu_{v}+k_{v}\right) \vec{\nabla} \times \vec{\omega}+k_{v} \vec{\nabla} \times \vec{N}=\vec{f}_{p m}
$$

To derive the pressure equation, depending on known velocity, vorticity and microrotation field, the divergence of equation (13) should be calculated, resulting in elliptic Poisson pressure equation:

$$
\Delta p-\vec{\nabla} \cdot \vec{f}_{p m}=0
$$

The advantage of the Boundary Domain Integral Method, originating from Boundary Element Method, lies in the application of Green's fundamental solutions as particular weighting functions. Different conservation models can be written with an appropriate selection of a linear differential operator $L[u]$ in the following general form:

$$
L[u]+b=0
$$

where the operator $L[u]$ can be either elliptic or parabolic, $u\left(r_{j}, t\right)$ is an arbitrary field function, and the nonhomogenous term $b\left(r_{j}, t\right)$ is applied for non-linear transport effects or pseudo body forces. 
The integral representation of flow kinematics (10) is derived from the elliptic Poisson partial differential equation, Škerget et al. [6], resulting in:

$$
c(\xi) v_{i}(\xi)+\int_{\Gamma} v_{i} \frac{\partial u^{*}}{\partial n} \mathrm{~d} \Gamma=e_{i j} \int_{\Gamma} v_{j} \frac{\partial u^{*}}{\partial n_{t}} \mathrm{~d} \Gamma-e_{i j} \int_{\Omega} \omega \frac{\partial u^{*}}{\partial x_{j}} \mathrm{~d} \Omega
$$

where $u^{*}$ is elliptic fundamental solution.

To apply integral representation based on diffusion-convection fundamental solution for flow vorticity (11) and microrotation (12), nonhomogenous velocity field $\vec{v}(r)$ must be decomposed into the constant $\vec{v}_{o}$ and a variable or perturbated part $\tilde{\vec{v}}(r)$. A general form of the diffusion-convective equation with first order chemical reaction term is therefore:

$$
\frac{\partial u}{\partial t}+\frac{\partial u v_{o j}}{\partial x_{j}}=a_{o} \frac{\partial^{2} u}{\partial x_{j} \partial x_{j}}-\kappa u-\frac{\partial \widetilde{v_{j}} u}{\partial x_{j}}+\frac{I_{o}}{c_{o}}
$$

Where $a_{o}$ and $c_{o}$ are constant transport material properties, $\beta$ is the reaction rate constant, while $I_{o}$ stands for known source term. For accumulation term $\partial u / \partial t$ the first order Euler scheme discretisation is used $\partial u / \partial t \approx\left(u_{F}-\right.$ $\left.u_{F-1}\right) / \Delta t$, where the subscript $F$ is indicating variable $u$ in current time step and subscript $F-1$ in previous time step and $\Delta t$ is the time step.

With use of equation (15) the equation (17) can be stated as:

$$
L[u]+b=a_{o} \frac{\partial^{2} u}{\partial x_{j} \partial x_{j}}-\kappa u-v_{o j} \frac{\partial u}{\partial x_{j}}+b
$$

where $L[\cdot]$ is linear differential operator, and $b$ stands for pseudo body force term. The parameter $\kappa$ is defined as a sum of time increment parameter from accumulation term discretisation and $\chi$ which accounts other reaction terms:

$$
\kappa=\frac{1}{\Delta t}+\chi
$$

If we suppose that we know the fundamental solution $u^{*}(\xi, s)$ satisfying the equation $L^{*}\left[u^{*}\right]+\delta(\xi, s)=0$, where $L^{*}[\cdot]$ denotes the adjoint operator to $L[\cdot]$ we can write:

$$
a_{o} \frac{\partial^{2} u^{*}(\xi, s)}{\partial x_{j}(s) \partial x_{j}(s)}-\kappa u^{*}(\xi, s)-v_{o j} \frac{\partial u^{*}(\xi, s)}{\partial x_{j}(s)}+\delta(\xi, s)=0
$$

The fundamental solution $u^{*}$ and its normal derivative is expressed as (Skerget et al [6]): 


$$
\begin{gathered}
u^{*}=a_{o} \frac{1}{2 \pi a_{o}} K_{o}(\zeta r) \exp \left(\frac{v_{o j} r_{j}}{2 a_{o}}\right) \\
\frac{\partial u^{*}}{\partial x_{j}} n_{j}=\frac{n_{j}}{2 \pi r^{2} a_{o}}\left[\zeta r K_{1}(\zeta r) r_{j}-\frac{r^{2}}{2 a_{o}} K_{o}(\zeta r) v_{o j}\right] \exp \left(\frac{v_{o j} r_{j}}{2 a_{o}}\right)
\end{gathered}
$$

where the parameter $\zeta$ is defined as:

$$
\zeta^{2}=\left(\frac{v_{o}}{2 a_{o}}\right)^{2}+\frac{\kappa}{a_{o}}=\left(\frac{v_{o}}{2 a_{o}}\right)^{2}+\frac{1}{a_{o} \Delta t}+\frac{\chi}{a_{o}}
$$

$K_{o}$ and $K_{l}$ are the modified Bessel functions of the second kind, $r_{j}(\xi, s)$ is the vector from the source point $\xi$ to the reference point $s$, while $r$ is its magnitude $r=\left|r_{j}\right|$ and $v_{o}^{2}=v_{o j} v_{o j}$.

By applying Green's theorem for scalar field function to equation (18) and applying the Gauss theorem to the domain integral of pseudo body source term $b$ which includes the convection for the perturbated velocity field only, source term and initial conditions from accumulation term discretisation, we can write:

$$
\begin{aligned}
c(\xi) u(\xi)+a_{o} \int_{\Gamma} u \frac{\partial u^{*}}{\partial n} \mathrm{~d} \Gamma & \\
& =a_{o} \int_{\Gamma} \frac{\partial u}{\partial n} u^{*} \mathrm{~d} \Gamma-\int_{\Gamma} u v_{o n} u^{*} \mathrm{~d} \Gamma+\int_{\Omega} \widetilde{v_{J}} u \frac{\partial u^{*}}{\partial x_{j}} \mathrm{~d} \Omega \\
& +\frac{1}{c_{o}} \int_{\Omega} I_{o} u^{*} \mathrm{~d} \Omega+\frac{1}{\Delta t} \int_{\Omega} u_{F-1} u^{*} \mathrm{~d} \Omega
\end{aligned}
$$

Equation (24) represents integral equation for kinetic of the property $u$. The meanings of $a_{o}, c_{o}$ and $I_{o}$ for each $u$ are listed in table 1.

Table 1: Generalised representation of kinetic equations in integral form.

\begin{tabular}{|c|c|c|c|c|c|}
\hline Equation & $u$ & $a_{o}$ & $\chi$ & $c_{o}$ & $I_{o}$ \\
\hline$(11)$ & $\omega$ & $\frac{\mu_{v}+k_{v}}{\rho}$ & 0 & $\frac{\rho}{k_{v}}$ & $\frac{\partial^{2} N}{\partial x_{j} \partial x_{j}}$ \\
\hline$(12)$ & $N$ & $\frac{\gamma_{v}}{\rho j}$ & $\frac{2 k_{v}}{\rho j}$ & $\frac{\rho j}{k_{v}}$ & $\omega$ \\
\hline
\end{tabular}




\section{Results}

For the test case the Poiseuille flow in the channel was chosen (Fig. 1). The mesh has 50 elements in the $x$ axis and 40 elements in $y$ axis. The mesh was refined in the vicinity of solid walls with refinement factor 4 . On the inlet the uniform velocity was prescribed $\left(v_{x=0}=1 \mathrm{~m} / \mathrm{s}\right)$ normal to the inlet boundary.

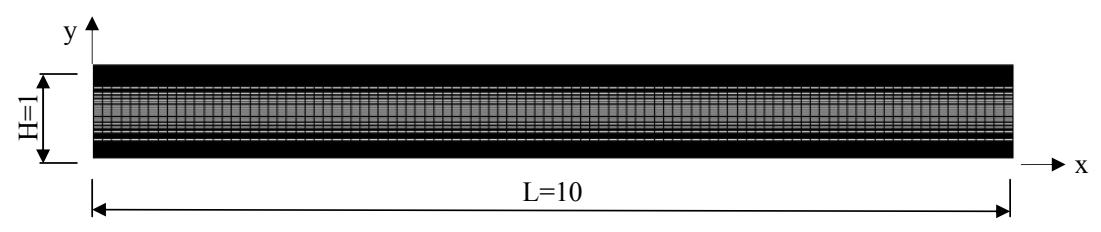

Figure 1: Geometry and mesh of the problem.

A general type of the boundary condition for the microrotation on the no slip walls for the 2D planar problem was proposed by Rees and Bassom [9]:

$$
N_{\text {wall }}=-n \frac{\partial v_{x}}{\partial y} \quad 0 \leq n \leq 1
$$

where $n=1 / 2$ indicates weakly concentrated suspensions (Jenna and Mathur [10]), $n=1$ indicates turbulent shear flows (Peddieson [11]) and $n=0$ is called strong concentrated suspensions (Guram and Smith [12]). Our calculation was performed for the $n=0$ and therefore microrotation was equal zero at the rigid boundaries. The initial velocity and microrotation fields were set to zero. Microinertia was defined as a square of the length scale $\left(j=L^{2}\right)$, as stated by the Rees and Bassom [9]. Viscosity gradient coefficient $\gamma_{v}$ or the spin gradient viscosity was taken in the form proposed by the Ahmadi [13] as:

$$
\gamma_{v}=\left(\mu+\frac{k_{v}}{2}\right) j
$$

The calculation was performed for different material parameters $K=0,0.1$, $0.5,10$ and 100 , representing the ratio between vortex viscosity and dynamic viscosity. The case $K=0$ was achieved by setting vortex viscosity to be zero. This case is presenting calculation of the micropolar fluid in which the microrotation does not affect the flow (as in the case of classical Navier-Stokes equation). On fig. 2 the microrotation profiles at the outlet are presented. An additional coefficient $\lambda$, which is a combination of vortex viscosity $k_{v}$ and spin gradient viscosity coefficient $\gamma_{v}$, is controlling the microrotation. When the parameter $k_{v}$ is zero also the coefficient $\lambda$ is zero. We can see that in the case $k_{v}=0$ also the coefficient $\lambda$ is zero and the microrotation is zero and that increasing of coefficient $\lambda$ is causing increase of microrotation. Results in fig 2 are consist with results of Eringen [1]. The pressure difference in dependence of the material parameter is shown in fig. 3 . 


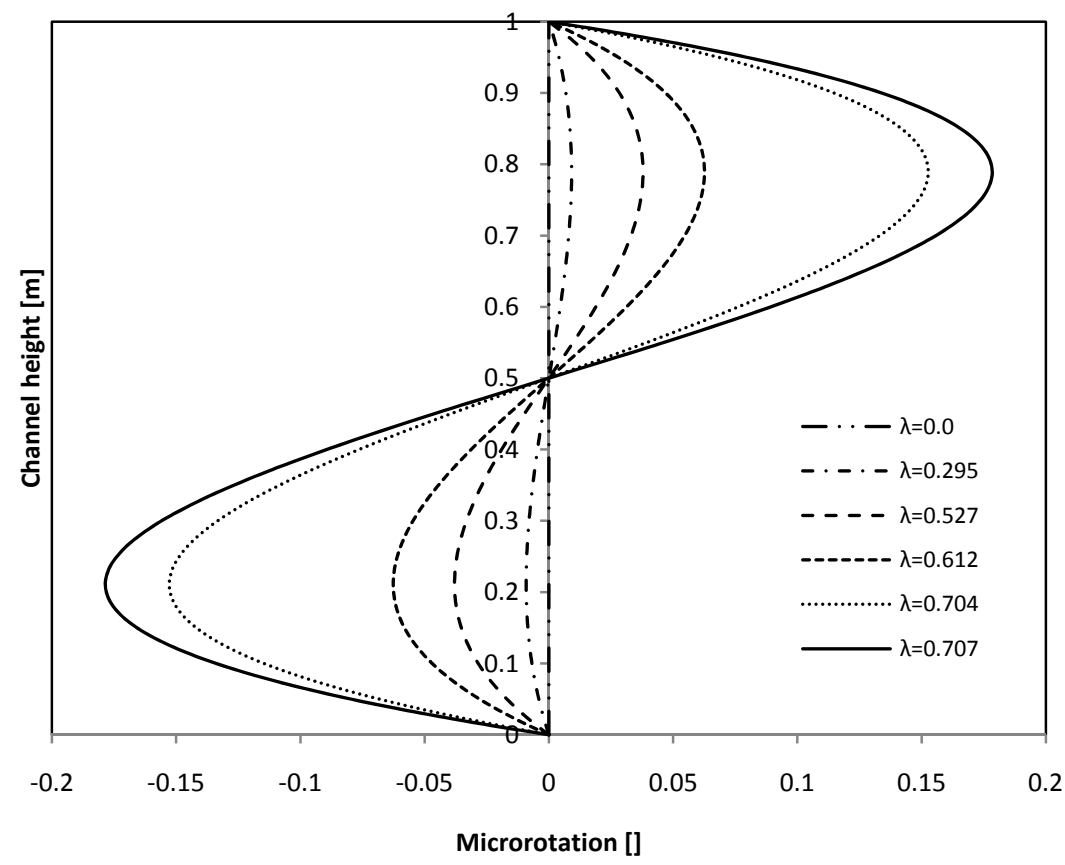

Figure 2: Microrotation profiles.

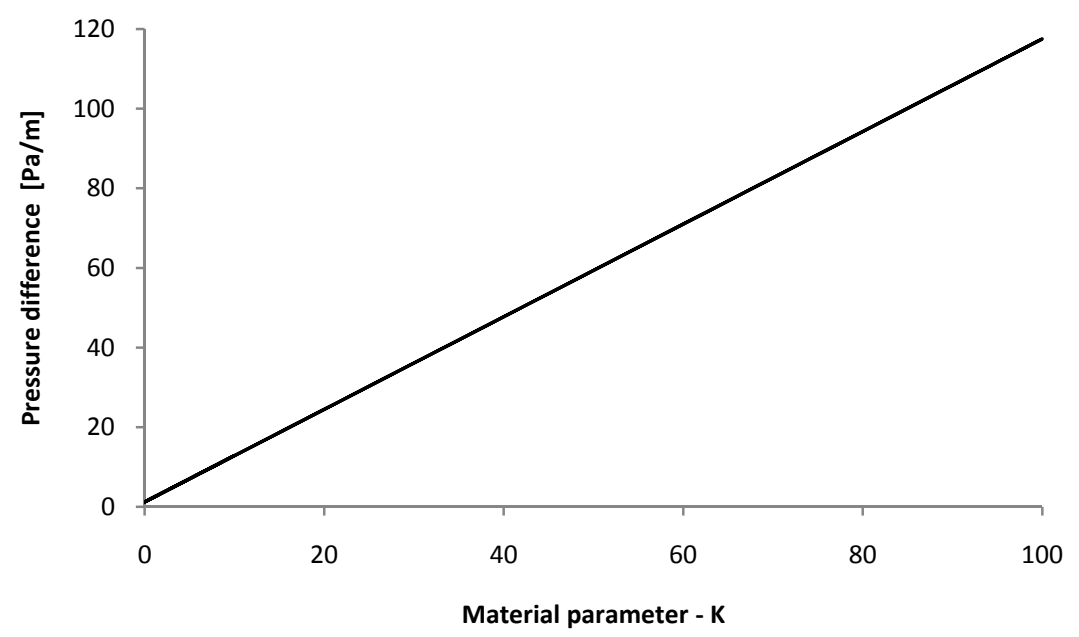

Figure 3: Pressure difference. 


\section{Concluding remarks}

The paper presented the derivation of differential as well as integral forms of equations in the framework of velocity-vorticity formulation for numerical simulation of fluid flow with micropolar fluid theory. The derivation showed that the derived equations include several additional terms, compared with the classical set of Navier-Stokes equations. The physical model was incorporated into the BEM numerical code. The derived numerical algorithm was used to analyze the effect of different parameters of micropolar fluid flow on flow in a channel. Computational results were also compared with results of other authors and comparison shows good agreement.

\section{References}

[1] Eringen, A.C., Theory of Micropolar Fluids. J Math Mech, 16, pp. 1-18, 1966.

[2] Eringen, A.C., Microcontinuum Field Theories: II. Fluent media. New York: Springer Verlag, 2001.

[3] Lukaszewicz, G., Micropolar Fluids: Theory and Application. Boston: Birkhäuser, 1999.

[4] Papautsky, I., Brazzle, J., Ameel, T., \& Frazier, A.B., Laminar fluid behaviour in microchannels using micropolar fluid theory. Sensors and actuators, 73(1-2), pp. 101-108, 1999.

[5] Pietal, A.K., Microchannels flow modelling with the micropolar fluid theory. Bulletin of the Polish Academy of Sciences, 52(3), pp. 209-214, 2004.

[6] Škerget, L., Hriberšek, M. \& Kuhn, G., Computational fluid dynamics by boundary-domain integral method. Int J Numer Met Engng, 46, pp. 1291$1311,1999$.

[7] Škerget, L., Hriberšek, M. \& Žunič, Z., Natural convection flows in complex cavities by BEM. Int J Numer Methods Heat Fluid Flow, 13(5/6), pp. 720-736, 2003.

[8] Hriberšek, M. \& Škerget, L., Boundary domain integral method for high Reynolds viscous fluid flows in complex planar geometries. Comput Methods Appl Mech Engrg, 194, pp. 4196-4220, 2005.

[9] Rees, D.A.S. \& Bassom, A.P., The Blasius boundary-layer flow of a micropolar fluid. Int J Engng Sci, 34, pp. 113-124, 1996.

[10] Jena, S.K. \& Mathur, M.N., Similarity solutions for laminar free convection flow of a hermomicropolar fluid past a nonisothermal flat plate. Int J Engrg Sci, 19, pp. 1431-1439, 1981.

[11] Peddieson, J., An application of the micropolar fluid model to the calculation of turbulent shear flow. Int J Engrg Sci, 10, pp. 23-32, 1972.

[12] Guram, G.S. \& Smith, C., Stagnation flows of micropolar fluids with strong and weak interactions. Comput Math Appl, 6, pp. 213-233, 1980. 
42 Boundary Elements and Other Mesh Reduction Methods XXX

[13] Ahmadi, G., Self-similar solution of incompressible micropolar boundary layer flow over a semi-infinite flat plate. Int J Engng Sci, 14, pp. 639-646, 1976. 